\title{
MAXIMILIANO CLADAKIS
}

\section{UNIVERSIDAD DE BUENOS AIRES - CENTRO DE ESTUdiOS FILOSÓFICOS - \\ ACADEMIA NACIONAL DE CIENCIAS DE BUENOS AIRES - \\ CONSEJO NACIONAL DE INVESTIGACIONES CIENTÍFICAS Y TÉCNICAS}

\section{EXISTENCIA Y ENCUENTRO CON EL OTRO}

JAN PATOCKA Y EL MOVIMIENTO DE LA VIDA

\section{EXISTENCE AND ENCOUNTER WITH THE OTHER. JAN PATOCKA AND THE MOVEMENT OF LIFE}

maxicladakis@yahoo.com.ar

Recepción: Abril 2019

Aceptación: Octubre 2019

\section{RESUMEN}

El objetivo del siguiente trabajo es abordar la manera en que la existencia humana significa ineludiblemente un encuentro con el otro en el pensamiento de Jan Patočka. En este sentido, la idea de "movimiento de la vida" resulta fundamental para la comprensión de una reconfiguración de la existencia y del sentido del encuentro con el otro en su discurrir en el mundo. La oposición "ser con" y "ser contra" se presentan como manifestaciones de un mismo proceso de cosificación que, a partir del "sacrificio", pueden ser superados en una dimensión más plena y auténtica de la existencia que es, al mismo tiempo el encuentro efectivo con el otro más allá de toda determinación funcionalista y utilitaria.

PALABRAS Clave

Patočka, existencia, intersubjetividad, fenomenología.

\section{ABSTRACT}

The aim of this essay is to address the way in which human existence inevitably means an encounter with the Other in Jan Patočka thought. In this sense, the idea of "movement of life" is fundamental for the understanding of a reconfiguration of the existence and of the meaning of the encounter with the Other in its course in the world. The opposition "being together" and "being against" are presented as manifestations of the same process of reification that, from the "sacrifice", can be overcome in a fuller and more authentic dimension of existence that is, at the same time, the effective encounter with the Other beyond all functionalist and utilitarian determination.

\section{KEY WORDS}

Patočka, existence, intersubjectivity, phenomenology.

$$
\text { Tábano, no. } 15 \text { (2019), 13-28 }
$$




\section{INTRODUCCIÓN}

El presente estudio tiene por objetivo abordar la manera en que el filósofo checo Jan Patočka comprende el encuentro con el otro como rasgo propio de la existencia humana. En el transcurso del trabajo las categorías de "ser-con" y de "ser contra" como así también la de "solidaridad" y la de "lucha" tendrán un rol sumamente relevante ya que, desde la perspectiva de Patočka, se trata de elementos correlativos que emergen en el segundo momento del movimiento de la vida. Precisamente, la idea de movimiento es nuclear en el abordaje patočkiano puesto que ella implica una reconfiguración del sentido tanto de la propia existencia como de la existencia del otro. En cada momento, acontece una conversión del sí mismo y de ese otro con quien soy en el mundo.

En este aspecto, consideramos que la circunscripción de estos elementos a uno de los momentos del movimiento de la vida significa una posibilidad de pensamiento sumamente enriquecedora en torno a la cuestión de la intersubjetividad. A lo largo de la filosofía contemporánea, los debates en torno a si el sentido originario de las relaciones con el otro es el del conflicto o el de la coexistencia han movilizado una serie de reflexiones determinantes para el desarrollo de la problemática, tal como lo evidencian, por ejemplo, las diferencias, en el campo de la fenomenología, entre Jean Paul Sartre, quien sostiene en El ser y la nada la primera posición, y Maurice Merleau-Ponty, quien afirma en la Fenomenología de la percepción la segunda idea..

A partir de ello, la relevancia del problema se abre en tres direcciones que se entrelazan dialécticamente. Por un lado, se trata de un aporte a los estudios patočkianos pues el encuentro con el otro y sus transfiguraciones en el despliegue de la vida son algunos de los ejes transversales que atraviesan la obra de Patočka. Por otro lado, la puesta en relación del planteo realizado por el filósofo checo con los debates dados dentro de la filosofía contemporánea, pueden ser útiles para brindar algunas herramientas que enriquezcan los estudios sobre el tema. En tercer lugar, la presentación de la solidaridad y de la lucha como el anverso y el reverso de una misma comprensión del otro dada en el dominio del rol y de la función, ofrecen una perspectiva que consideramos sumamente original.

Con esta finalidad, el trabajo se estructurará en cuatro apartados. En el primero de ellos, se abordará la manera en que Patočka comprende la relación entre "mundo" y "existencia" de una forma en qué esta no puede ser demarcada a partir de los criterios de objetividad característicos de la ciencia moderna. En el segundo, expondremos la problemática acerca del sentido del encuentro originario con el otro a partir de la oposición "ser-con/ser-contra" en el decurso de la filosofía contemporánea, esencialmente en la fenomenología post-husserliana. El tercer apartado tendrá como eje, la relación que establece Patočka entre la lucha contra el otro y el dominio del trabajo y de las capacidades laborales que distinguen al segundo 
momento del movimiento de la vida. En el cuarto y último apartado, se expondrá la posibilidad de superación de la lucha a partir del sacrificio como momento donde la existencia se recupera a sí misma y realiza un encuentro efectivo con el otro más allá de las mediaciones funcionalistas.

\section{EXISTENCIA Y MUNDO}

Según Patočka, la existencia posee un carácter indeterminado si comprendemos la determinación a partir de criterios lógico-objetivos. La pregunta acerca de ella se torna dificultosa, carente de la posibilidad de una respuesta precisa y univoca. En “¿Qué es la existencia?”, el filósofo checo señala que esta difícultad radica tanto en el fenómeno mismo de la existencia como en la forma en que las preguntas acerca del "qué es" se circunscriben, en la época contemporánea, a los criterios de validación propios de la ciencia moderna. "¿Cómo pensar la existencia, cómo hablar de ella si la esencia de un concepto reside justamente en la objetividad que el pensamiento fija, de suerte que todos pensemos algo idéntico y no ya sólo algo igual?". ${ }^{1}$

El planteo patočkiano remite a las reflexiones de aquello que puede ser denominado como "existencialismo" o "filosofía de la existencia": ${ }^{2}$ la imposibilidad de reducir la existencia a un elemento dentro de un sistema de pensamiento determinado por los principios de la exactitud y del cálculo. Ni la existencia puede ser reducida a una formulación científica ni la ciencia, en su sentido moderno, es capaz de abordarla. Si "la ciencia entera con toda su claridad y potencia, descansan sobre este hecho: pensar lo idéntico", ${ }^{3}$ la existencia es un fenómeno imposible de abordar por ella en su totalidad.

La existencia implica el vivir en distintos planos que se encuentran "atravesados por el perderse a sí mismo, por el buscarse a sí mismo y, en ocasiones, también por el encontrarse el hombre a sí mismo". ${ }^{4}$ Estos planos, por lo tanto, hacen al ser de la existencia. En ellos, la existencia se despliega y se hace ser, se pierde y

\footnotetext{
${ }^{1}$ PAtočKa, J., El movimiento de la existencia humana, trad. T. Padilla, J. M. Ayuso y A. Serrano de Haro), Ediciones Encuentro, Madrid, 2004, 48.

2 De aquí en adelante, para evitar posibles malos entendidos, emplearemos el término "fílosofía de la existencia" en lugar de existencialismo siguiendo el planteo de Merleau-Ponty en su conferencia "La filosofía de la existencia". En ella el autor distingue entre "existencialismo" y "filosofía de la existencia", caracterizando al primero como el movimiento filosófico francés que se da en los años de posguerra encabezado principalmente por Sartre, mientras que el segundo tendría un espectro más amplio que abarcaría a autores como Kierkergaard y Heidegger (Cf., MERlEAU-PonTY, M., Parcours deux 19511961, Lagrasse, Verdier, 2000).

${ }^{3}$ PATOČKA, El movimiento de la existencia humana, 48.

${ }^{4}$ Ibid.
} 
se encuentra, no se trata de planos sobre los que transcurre como si fuera un elemento ya acabado. La existencia se determina a sí misma en este tránsito.

Patočka comprende como uno de los elementos fundamentales de la existencia el hecho de ser "vida en la verdad" y sostiene que esto significa "consecuencias de muy notable alcance para la comprensión del hombre, para su posición en el Universo, para su relación con los demás y consigo mismo". ${ }^{5}$ Desde la perspectiva de la existencia, la verdad no es comprendida como correspondencia o adequatio. El filósofo checo comprende la verdad en un sentido más originario que el del juicio. Este sentido originario compromete al hombre en su totalidad.

No caben dudas de que el planteo patočkiano tiene una notoria correspondencia con la aletheia heideggeriana y, también, con la iluminación sartreana (la cual a su vez se encuentra influenciada por la primera). Si la problematización de la existencia significa un giro en cierta forma de comprender la filosofía, dicho giro es también un giro con respecto a la comprensión de la verdad. La relación entre existencia y verdad no es la de la posesión, sino la de "estar en la verdad". Las palabras de Patočka son muy claras:

Estar en la verdad quiere originalmente decir «interesarse por», «inclinarse por» una de mis posibilidades esenciales, o sea, no ser indiferente a lo que soy y a cómo lo soy. Sólo merced a esta no-indiferencia y en el seno de ella puede un ser que es en sí mismo una parte del Universo existir a la vez en la verdad. Cabe decir incluso que el interés por el ser propio, por sus posibilidades esenciales, es conditio sufficiens de todo estar en la verdad. Para que un ser exista en la verdad no tiene, pues, que salirse de las conexiones que forman el Universo de cosas existentes, no tiene que colocarse fuera del Universo, no tiene que «contener en sí mismo al sujeto trascendental de toda posible experiencia». Basta con que tenga la capacidad de proyectar el mundo en el sentido arriba indicado, «capacidad» ésta que no es distinta de la propia realidad de este ser, de su ser propio. ${ }^{6}$

En este sentido, "contrariamente al significado original de verdad como descubrimiento, la teoría de la verdad como la correspondencia hace que la verdad sea meramente una cosa entre otras cosas". ${ }^{7}$ En su sentido originario, por el contrario, la verdad abraza la existencia en su relación integral con el mundo. La existencia que es en la verdad es aquella que se encuentra comprometida con sus posibilidades concretas en el mundo. Existencia, mundo y verdad se articulan en un mismo plexo. Las conceptualizaciones aisladas de ellos forman parte de las abstracciones propias

\footnotetext{
${ }^{5}$ Ibid., 58.

${ }^{6}$ Ibid., 79.

${ }^{7}$ SUVÁK, V., "The Essence of Truth (aletheia) and the Western Tradition in the Thought of Heidegger and Patocka", en: Thinking Fundamentals, IWM Junior Visiting Fellows Conferences, Vol. 9, Vienna, 2000 .
} 
del entendimiento formal. La propuesta del filósofo checo remite inexorablemente a las tesis fenomenológicas acerca de la búsqueda del sentido originario de la existencia, sentido anterior a las formulaciones llevadas a cabo por las ciencias modernas.

Patočka retoma los planteos de Husserl y de Heidegger acerca del mundo como horizonte donde discurre la vida. Las siguientes palabras del filósofo checo expresan con claridad su propuesta.

El mundo en sentido originario no es la reunión total de las cosas que existen, sino la conexión de sentido que tiene ante sí una vida humana que se está realizando a sí misma, o sea, que se comprende activamente a sí misma. El mundo no es nada real, no está hecho de cosas ni de relaciones puramente reiformes entre cosas. Pero tampoco es vida ni vivencia. El mundo es un tercero, algo esencialmente distinto del ente, que, sin embargo, muestra y desvela lo que son las cosas y cómo lo son. Las conexiones del mundo están abiertas en origen gracias a una posibilidad fundamental de mi propia vida: las cosas se muestran en virtud de su poder estar a la mano o a contramano, en relación justamente con esta posibilidad de servir para o de obstaculizar; tal relación yo no la comprendo por convertirla en objeto para mí, sino por el hecho de que yo soy esta posibilidad, de que yo la realizo. ${ }^{8}$

\section{LA APARICIÓN DEL OTRO: DEL MITTSEIN AL SER PARA-OTRO}

El mundo es el horizonte sobre el cual la existencia discurre y donde los entes se abren. Sin embargo, el mundo no es el horizonte de una sola existencia. El mundo es donde me encuentro con los otros. Ser en el mundo significa ser con los otros. El encuentro con el otro irrumpe, entonces, como problema nodal dentro de una filosofía que intenta comprender la existencia en su radicalidad originaria.

En algunos pensadores modernos, la reflexión acerca de la existencia del otro tiene como correlato la problemática acerca de los criterios de validación de dicha existencia. Este último punto, heredero de la reflexión cartesiana, es descartado por la filosofía de la existencia. La validación de la existencia del otro se encuentra articulada con el problema del solipsismo el cual "es un falso problema". En cierta medida, la demostración de la existencia del otro se relaciona con la importancia de la demostración en un sentido más amplio y en la búsqueda de un método que pueda instituir una ciencia universal. En el planteo cartesiano, es absolutamente lógico que deba demostrar la existencia del otro ya que, antes tuve que demostrar mi propia

\footnotetext{
${ }^{8}$ PATOČKA, El movimiento de la existencia humana, 79.

${ }^{9}$ Merleau-Ponty, M., Phénoménologie de la perception, Gallimard, París, 403.
} 
existencia. Sin embargo, "Descartes no ha probado su propia existencia. Pues, en efecto, yo siempre he sabido que existía, no he cesado jamás de practicar el cógito". ${ }^{10}$

Desde la perspectiva de una filosofía que intenta expresar la existencia desde su concretitud originaria, la idea de demostración es superflua: yo existo, el otro existe, el mundo existe. Esto no implica una resolución de la problemática, sino un cambio de dirección. El problema de la existencia del otro no es el de su validación gnoseológica, sino el de su sentido. En este aspecto, el planteo de Patočka se inscribe con claridad dentro de la tradición fenomenológica. El retorno al mundo de la vida propuesto por Husserl y seguido por sus continuadores significa, entre otras cosas, un cambio de perspectiva donde la pregunta por el sentido adquiere una importancia nodal. La vida se comprende, entonces, como vida orientada hacia una dirección, como vida orientada a un fin. El otro existe, es un factum al igual que lo es mi existencia. Ahora bien, la cuestión es qué significa la existencia del otro y cual es el sentido originario de mi relación con él en un mundo compartido.

Como se verá en las páginas siguientes, los tres momentos en los que discurre el movimiento de la vida significan, entre otras cosas, reconfiguraciones del sentido de las relaciones con el otro. De la misma manera en que otros aspectos, la propuesta patočkiana retoma los planteos formulados por pensadores anteriores o contemporáneos para, desde esa reasunción, desplegar su propio pensamiento. En este punto, los debates acerca del sentido originario de la relación con el otro constituyen el suelo sobre el que Patočka esbozara sus ideas.

En este sentido, la noción heideggeriana de Mitsein es una idea indudablemente relevante en la filosofía del siglo XX. Se trata de uno de los existenciarios constitutivos del Dasein en la filosofía de Heidegger. En Ser y tiempo, ser-en-el-mundo es ser con-los-otros. El planteo heideggeriano no necesita de ningún tipo de verificación de la existencia del otro, puesto que, en la estructura ontológica de la existencia, ya se encuentra involucrado el otro. No es del interés del presente trabajo ahondar y problematizar en el pensamiento de Heidegger con respecto a la idea del ser-con. Lo que sí nos convoca es la forma en que este es interpretado para el posterior despliegue del problema.

A partir del planteo heideggeriano, dentro de la fenomenología se dará un interesante debate acerca del sentido del encuentro con el otro. En este aspecto, la filosofía de Sartre puede ser comprendida como el polo antagónico ya que, para el filósofo francés, el "ser-con" no tiene un sentido originario, sino que lo que determinará las relaciones con el otro será el conflicto, el "ser-contra". Precisamente, Dan Zahavi señala la diferencia entre ambos planteos ya que "según Sartre, el concepto de Heidegger de ser-con (Mitsein), falla completamente en capturar nuestra

${ }^{10}$ SARTRE, J.P., L'être et le néant. Essai d’ontologie phénoménologique, Gallimard, París, 1943, 269. 
relación original y fundamental con los otros". ${ }^{11}$ En El ser y la nada Sartre asume el planteo heideggeriano acerca de que la relación con el otro es una relación de ser frente a los planteos trascendentales. Sin embargo, para el filósofo francés, el Mitsein "no puede constituir una estructura ontológica de la realidad humana". ${ }^{12}$ Mientras el ser-con indica que el sentido originario de mi relación con el otro es el de equipo, el ser para otro supone que lo originario es el conflicto.

En la fenomenología francesa de posguerra, la relación entre intersubjetividad y conflicto es sumamente importante. La problemática no es meramente teórica, sino que se entrecruza con los acontecimientos histórico-políticos de la época. Como lo señala el título del notable texto de Merleau-Ponty, "La guerra tuvo lugar", Francia fue ocupada, luego liberada, el comunismo se hizo una realidad efectiva y conllevó a regímenes tanto o más brutales que aquellos que decía combatir. Los optimismos filosóficos vieron derrumbarse sus premisas. En efecto, había una "filosofía optimista, que reducía la sociedad humana a una suma de conciencias siempre dispuestas para la paz y la felicidad", mientras que se sabía que "que existían campos de concentración, que los judíos eran perseguidos". ${ }^{13}$ Merleau-Ponty mismo se responsabiliza de haber formado parte de ese grupo de intelectuales que sostenía un pensamiento contrario a la realidad que efectivamente se encontraba atravesando Europa.

Sin embargo, el planteo de Merleau-Ponty se diferencia del de Sartre. Si el autor de El ser y la nada comprende el conflicto como el sentido originario de las relaciones con el otro, Merleau-Ponty habla de una coexistencia originaria y retoma el concepto husserliano de Ineinander, ser uno en el otro. Igualmente, el planteo de Merleau-Ponty no significa una anulación del conflicto ni tampoco la traslación de este a un fenómeno derivado. Cuando el fenomenólogo francés habla de esta coexistencia originaria articula la noción de reciprocidad con la de conflicto. En la Nota a Maquiavelo, Merleau-Ponty presenta dos ideas sumamente interesantes. La primera se trata de una construcción semántica en cuyo sentido paradojal habita una idea sumamente sugerente: "Comunión de Santos negra". ${ }^{14}$ La segunda es la rotunda sentencia acerca de que "la vida colectiva es el infierno". ${ }^{15}$

Ambas ideas se articulan en una dialéctica donde el reconocimiento y el conflicto se entrelazan en una comprensión de la intersubjetividad que sobrepasa tanto el punto de partida de la primacía del sujeto particular como el de una suerte

11 Zahavi, D., "Intersubjectivite in Sartre's Being and Nothingness" en: Alter. Revue de Phénoménologie. Sartre fénoménologue, Editions Alter, Paris, 2002, 266.

${ }^{12}$ SARTRE, L'être et le néant. Essai d'ontologie phénoménologique, 485.

${ }^{13}$ Merleau-Ponty, M., Sens et non-sens, Nagel, Paris, 1963, 268.

${ }^{14}$ Merleau-Ponty, M., Signes, Gallimard, Paris, 1960, 268.

15 Ibid., 270. 
unidad homogénea primordial. La "Comunión de Santos negra" designa un reconocimiento originario puesto que hablar de comunión exige la necesidad de lazos de reconocimiento y de reciprocidad. Sin embargo, dichos lazos llevan en sí el antagonismo, el conflicto y la violencia. La dimensión infernal de la vida colectiva se manifiesta sobre este horizonte de comunión originaria.

\section{SOLIDARIDAD Y LUCHA: EL MOVIMIENTO DE LA VIDA}

Entre el planteo de Patočka y el de Merleau-Ponty hay varios puntos de encuentro. Para ambos, el conflicto y la lucha son el anverso del reconocimiento. El debate acerca de si el ser-contra es previo al ser-con, o viceversa, queda salvado a partir de la comprensión de que se trata de fenómenos que corren en paralelo. Sin embargo, en Patočka la idea de movimiento especifica el modo en el cual se da esta relación.

Esta idea atraviesa varias de sus obras. La vida humana o existencia es definida a partir del movimiento. Sin embargo, no se trata de la dinamis aristotélica la cual "es un cambio en el dominio de unos contrarios que están dados: un color sólo puede pasar a ser otro color, un sonido sólo puede cambiar a otro sonido, una sustancia inanimada a una animada y al revés". ${ }^{16}$ Según Patočka, "el movimiento de la existencia es [...] el proyecto de las posibilidades en cuanto realización de ellas, no son posibilidades que estuvieran dadas de antemano en algún ámbito previo determinante del sustrato". ${ }^{17}$

Para Patočka, el movimiento de la vida es un movimiento de autodeterminación de la propia vida que no responde a determinaciones externas. La vida es su propio movimiento que, en su despliegue, realiza posibilidades que emergen de su propia libertad. Este movimiento no responde, por tanto, a leyes de causalidad sino a la libertad de la existencia misma. Cómo señala Iván Ortega Rodríguez, "Patočka piensa que este movimiento de transformación y advenimiento de propiedades, en el sentido más radical y propio de autorrealización, es el que mejor explica la trama íntima de la existencia humana como realización de sí asumiendo posibilidades". ${ }^{18}$

Ahora bien, para Patočka, este movimiento se encuentra constituido por tres momentos. El primer momento es el del arraigo originario en el mundo, el segundo es el del dominio del trabajo y de la función donde nos alienamos en el rol que nos es otorgado por la división del trabajo, el tercero se refiere al sacrificio donde

\footnotetext{
${ }^{16}$ PATOČKA, El movimiento de la existencia humana, 81.

17 Ibid.

${ }^{18}$ ORTEGA RodRíGUEZ, I., "Existencia humana, mundo y responsabilidad en la fenomenología de Jan Patočka”, Investigaciones Fenomenológicas, vol. Monográfico 4/I : Razón y vida, 2013, 252.
} 
alcanzamos la autenticidad de nuestra existencia por medio del despojamiento de todo ser dado en el momento anterior. Cada momento se entrelaza con el otro en una dialéctica donde cada uno de ellos implica una reconfiguración del anterior, lo que implica una reconfiguración de la vida en su sentido íntegro, donde se reconfigura también la relación con el mundo y con los otros.

Es en el segundo momento de este movimiento donde el filósofo checo inscribe la relación solidaridad-lucha. Precisamente, este momento aparece signado bajo el dominio del trabajo y de las capacidades laborales. Al tratarse de una dialéctica existencial, este momento emerge del anterior. Cómo se ha mencionado, el primer momento del movimiento de la vida es el de ser acogido por la tierra. Ella es lo que nos antecede y que nos dota de una historia previa. Somos arrojados a la vida y la tierra nos acoge brindándonos un pasado previo a nuestra emergencia en el mundo.

En "El mundo natural y la fenomenología". Patočka señala que:

La relación de enraizamiento surge de la dimensión del pasado. Es una relación con aquello que siempre esta ahí una relación que penetra, a través de las estructuras humanas, hasta el cielo y la tierra, hasta la totalidad previa, y cuyo dominio es la conciencia previa de horizonte y la relación original con la totalidad, la disposición de humor, el sentimiento de la situación original. ${ }^{19}$

La acogida de la tierra se abre como un pasado que nos recibe en el mundo. Patočka compara esta instancia con la infancia en la cual se constituye el ser propio a partir del contacto con los padres, los hermanos, etc., lo cual genera la adopción de un "mundo". La tierra, por lo tanto, se encuentra constituida como un suelo intersubjetivo y como una cultura. La tierra es en los otros. Enraizarse en la tierra es enraizarse en los otros, ser aceptado y acogido por ellos. "En los otros la tierra se vuelve así calurosa, clemente, acogedora". ${ }^{20}$ La tierra es suelo de arraigo ya que ella es el ser recibido por los otros. En el primer momento del movimiento de la vida, los otros son quienes nos acogen y nos dan un arraigo en el mundo. La dimensión temporal que prima es el pasado. Somos herederos de un pasado que antecede a nuestra llegada al mundo. En este aspecto, nos encontramos definidos a partir de la pasividad, recibimos una lengua, una serie de valores, etc.

Sin embargo, la tierra, al ser tierra nutricia, necesita ser trabajada. Esta necesidad de "trabajar la tierra" arroja a la existencia al segundo momento de su movimiento. Aparece el dominio del trabajo bajo el imperativo de preservar la vida. Esto significa una transformación del otro, de ser arraigo pasa a ser fuerza copartícipe de una empresa común. "El otro es aquí la fuerza coparticipe que me es indispensable

\footnotetext{
${ }^{19}$ PATOČKA, El movimiento de la existencia humana, 31.

${ }^{20}$ Ibid., 47.
} 
y es una individualidad, una cosa entre otras cosas como yo mismo lo soy en esta esfera". ${ }^{21}$

La coparticipación, la co-operación y el consumo compartido son las formas fundamentales de la relación con el otro. Estas formas establecen la cosificación del otro, al mismo tiempo que mi propia cosificación. El otro y yo aparecemos como medios para una tarea común dentro de la cual somos indispensables. Sin embargo, esta indispensabilidad se da desde criterios de utilidad. Tanto el otro como yo mismo nos transmutamos en nuestras funciones, en nuestros papeles, en nuestras capacidades. Esta transmutación implica la pérdida del sí mismo en el rol y la función. Por tanto, cada uno es intercambiable, lo que, en todo caso, no es intercambiable es el rol o la función que llevamos a cabo. "En el dominio del trabajo y de las destrezas laborales somos intercambiables, ya que este dominio proviene del hacer, del comercio con los distintos poderes en su fragmentación, en las circunstancias individuales, en la ocasión particular". 22

Si en el primer momento del movimiento de la vida, la dimensión temporal que prima es el pasado, en este segundo momento, lo hace el presente. El presente domina la vida a través del "hacer". En esa dimensión temporal mi relación con el otro es una relación de exterioridad. No me relaciono con el otro sino en virtud a su hacer, un hacer, cabe destacar, que se encuentra circunscripto a la designación de roles, no se trata de un hacer libre y auténtico que involucra la totalidad e integridad de mi persona, sino que, por el contrario, se realiza en la fragmentación de ella. A partir de esta primacía del presente, la vida se reproduce a sí misma a partir de la repetición. En el mundo del trabajo, el tiempo es sucesión serializada de "ahoras", circularidad perpetua del presente.

Precisamente, es bajo esta temporalidad, que mi relación con el otro no se agota en la del "ser-con" sino que emerge la dimensión del "ser-contra".

Es un movimiento caracterizado, en lo que concierne a la relación con los otros, por la misma atomización que representa la dispersión en los instantes, en un presente iterativo. El coparticipante en esta esfera es un partenaire que constituye un centro cerrado para sí, que se dirige solo y por sí hacia la prolongación de (desprendimiento) de sí mismo. ${ }^{23}$

La rivalidad y la lucha emergen de las mismas necesidades que gestan la solidaridad. "Los copartícipes son siempre, en sentido rivales, aunque se presten ayuda, compartan, etc.". ${ }^{24}$ La transmutación de la vida en una fuerza determinada por

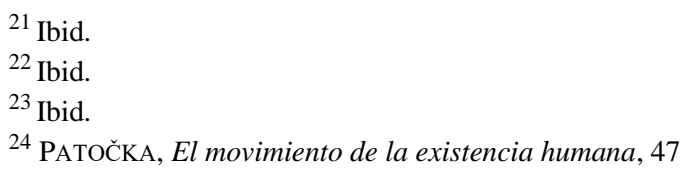


el criterio de la utilidad instituye el sentido mismo de la lucha. Trabajamos en común la tierra, sin embargo, lo hacemos para facilitar la satisfacción individual. En ese trabajo común el otro es un medio para mi interés individual y yo soy lo mismo para él. Bajo el dominio del trabajo, se produce una igualación de la vida. La reducción de la vida a fuerzas coparticipes instituyen la posibilidad de la solidaridad en términos utilitarios y la de la lucha.

El ser coparticipes en el trabajar la tierra es ser "coexplotadores". La finalidad de la explotación no es otra que la perpetuación de la propia vida: yo para el otro y el otro para mí podemos ser objeto de esa explotación. La ubicación de la lucha en el segundo momento del movimiento de la vida llevada a cabo por Patočka señala que esta se da en un ámbito de homogeneización y de despersonalización de la vida. Reciprocidad y lucha forman parte de un mismo plexo donde el mundo es interpretado bajo la perspectiva del funcionalismo mecanicista. Somos fuerzas a emplear en la preservación de la vida. De esta manera, estas fuerzas pueden obrar de manera conjunta o una contra la otra.

La misma comprensión del otro como fuerza lleva consigo la cosificación del otro. La designación tiene un marcado carácter fiscalista-mecanicista. "Las relaciones de fuerza presuponen una mirada llevada sobre el hombre del exterior, que lo ve como una fuerza, un auxiliar o un obstáculo en la aproximación de los medios cósicos en vista del fin que es la vida”. La transmutación del otro y de mí mismo en fuerza significa que puede tratarse tanto de un auxilio como de un obstáculo. En el dominio del trabajo, la fuerza es un medio para un fin. Para cada uno: la finalidad es distinta ya que todo obrar tiene como miras la preservación de la propia vida. La atomización de la vida, hace de cada uno el fundamento de todo obrar.

Sin embargo, en este punto radica la paradoja. Muy lúcidamente, Patočka observa que, al ser cada uno el centro y la finalidad del obrar, se realiza una igualación entre todos los individuos. Al ser el fundamento del obrar la propia preservación, el interés personal se presenta como interés general.

Siguiendo su interés personal (que es lo más general en lo que todos acuerdan), los distintos centros individuales se revelan como fuerzas junto a otras fuerzas, como realidades fragmentadas y en continuo movimiento de coalición, de influencia de unas sobre otras, de frenarse unas a otras, y así siempre de nuevo y en sempiterna actualidad. ${ }^{25}$

\section{SACRIFICIO Y AUTENTICIDAD}

Cómo se ha señalado en páginas anteriores, la cuestión del conflicto y de la lucha es una problemática sumamente importante en la filosofía contemporánea. De

25 Ibíd. 
igual manera, lo es la cuestión en torno a su posible superación. En Hegel, la dialéctica del señor y del siervo se resuelve en una nueva figura del Espíritu; en Marx a partir de la abolición de la propiedad privada y de la emergencia del comunismo como traspaso del Reino de la Necesidad al Reino de la Libertad; en Sartre, no habría resolución puesto que el conflicto es constitutivo de la estructura ontológica del ser para-sí. Por su parte, en Merleau-Ponty si bien el conflicto no puede ser anulado, debe ser canalizado institucionalmente.

En el pensamiento de Patočka, la posibilidad de la superación de la lucha sólo se da en la superación del segundo momento del movimiento de la vida. Es decir, en la lucha entre fuerzas no existe la posibilidad de superación del conflicto. Es en el tercer momento del movimiento de la vida, donde acontece la superación de la relación de rivalidad. Esto significa que en el campo mismo de la lucha no existe la posibilidad de superación de ella. En este punto, Patočka se distancia de Hegel. "Hegel analizó dialécticamente esta situación en el sentido de combate a muerte. Pero tal lucha no engendra ninguna autoconciencia positiva, dado que el otro era aquí tan destructivo y negativo como yo mismo". ${ }^{26}$

Al surgir la lucha de la pérdida de sí en el rol, ella no puede representar una superación de la lucha ni de la pérdida. La relación que establece Patočka entre ambos fenómenos torna necesaria una transfiguración integral de la vida. Esta transfiguración se da en el tercer momento del movimiento de la vida.

Este momento surge del anterior y se da por medio de una reconfiguración del sentido de la lucha. La lucha deja de ser contra el otro y se presenta en su sentido más profundo. El hombre se enfrenta con "la finitud, con la nada y con la muerte". ${ }^{27}$ El otro, pues, deja de ser el antagonista del enfrentamiento. El rol, que es el fundamento de la lucha en el segundo momento del movimiento de la vida, se muestra aquí como superfluo. El enfrentamiento nuclear de la vida sacude "todo rol".

Sin embargo, parte de un rol que emerge en el momento anterior. La lucha y la rivalidad nos convierten en combatientes, ahora bien la figura del combatiente adquiere su modo más auténtico.

Puede decirse que el combate y la condición de combatiente en sentido externo da al hombre ocasión de asir y comprender la lucha en el sentido verdadero y profundo. El combate en este segundo sentido significa la conquista de lo que hay en nosotros, de la dispersión de los instantes sucesivos de un presente alienante el distraerse en lo externo cabe lo cual se está. ${ }^{28}$

\footnotetext{
26 Ibíd., 44.

27 Ibíd., 48.

28 Ibíd., 49.
} 
En el primer apartado se hizo mención a la existencia como "vida en la verdad". La pérdida del hombre en el rol significa, entre otras cosas, la caída de ese estar en la verdad. En el momento de enfrentamiento con el no ser, la vida se recobra a sí misma en su autenticidad. En un entramado de lenguaje paradojal, Patočka señala que este conquistarse a sí mismo, es, al mismo tiempo, una perdida de sí. En este momento, la existencia se consolida en su autenticidad pues asume su propia finitud. $\mathrm{Si}$ "estar en la verdad" es encontrarse comprometido con las propias posibilidades, la existencia que se asume en su finitud regresa a la verdad, tras su pérdida en el rol.

En "El hombre espiritual y el intelectual", Patočka habla de la vida espiritual como "vida a la intemperie". ${ }^{29}$ Precisamente, en el dominio de la función, el mundo se presenta como evidente y no-problemático. La vida que emerge en el tercer momento del movimiento acepta que no hay nada de estable ni de seguro. A partir de la emergencia de esa nueva vida "no podemos aceptar nada como consumado y dado" y "todo lo que habíamos aceptado como evidente, no es evidente"30. La aceptación de nuestra finitud se da en paralelo con la aceptación de la contingencia originaria del mundo.

El tercer momento del movimiento de la vida es el de la superación del dominio del trabajo y de la alienación, y es el futuro el que define su temporalidad. En este momento, se revela la existencia auténtica del hombre la cual se abre hacia una trascendencia que se halla más allá del mundo del trabajo y que, también, implica un trans con respecto a la tierra como arraigo originario. En las "Notas sobre la prehistoria de la ciencia del movimiento: el mundo, la tierra, el cielo y el movimiento de la vida humana", Patočka señala:

El hombre aquí descubre su existencia no como aceptada y enraizada, sino en su desnudez $-\mathrm{y}$ descubre en el mismo instante que la tierra tiene un trans—, un más allá. Eso quiere decir también que no hay en ella nada que pueda dar a la existencia un apoyo definitivo, un objetivo final, un por qué válido de una vez por todas. ${ }^{31}$

Patočka observa que la obra que se consolida aquí es la muerte. Sin embargo, la muerte no es la negación de la vida, sino que implica el enriquecimiento de ella. Este movimiento que la vida realiza sobre sí misma, por lo tanto, no debe comprenderse como una incursión en el nihilismo. Por el contrario, en la asunción de la finitud y de la muerte, la existencia se abre hacia un "más alto" y, en ese giro, la finitud se rebasa a sí misma hacia la infinitud.

\footnotetext{
${ }^{29}$ PAтOČKA, J., Libertad y sacrificio, trad. de I. Ortega Rodríguez, Sígueme, Salamanca, 2007, 256.

${ }^{30}$ Ibíd., 251.

${ }^{31}$ PATOČKA, J., "Notas sobre la prehistoria de la ciencia del movimiento: el mundo, la tierra, el cielo y el movimiento de la vida humana", Intentum. Cuadernos de gnoseología, trad. de D. Maffia, Universidad de Buenos Aires, CABA, 1996, 33.
} 
Al convocar la finitud y adherirse a ella acontece algo distinto que al proclamar la nada y el nihilismo. En esta adhesión tiene lugar un auténtico rebasamiento de la finitud, en el sentido de superarla y conservarla. La vida no sólo es capaz de prolongarse a base de ir perdiéndose, sino también de transformarse por la renuncia de sí. ${ }^{32}$

En este aspecto, la figura del "sacrificio" aparece como un elemento fundamental dentro del planteo patočkiano. El filósofo checo establece una profunda relación entre esta figura y la de la autenticidad. Es por medio del sacrificio de sí que la existencia recobra su verdad. Sin embargo, cabe aclarar que cuando Patočka habla de "sacrificio" no se refiere a un fenómeno que puede ser circunscripto a la lógica de recursos empleables para un fin, sino que se trata, ante todo, de un "sacrificio por nada, si entendemos con nada todo lo que no es ningún ente". ${ }^{33}$

En ese "por nada" acontece la apertura a un "trans" de la que tanto el cielo como la tierra se muestran como anuncios. Como señala Aviezer Tucker, en el sacrificio se "descubre la nada como una apariencia bajo la cual el ser muestra su diferencia con los seres dentro del mundo". ${ }^{34}$ El sacrificio se presenta como una vía de acceso al ser. La diferencia heideggeriana entre ser y ente se despliega en el filósofo checo como la posibilidad de superar el dominio del rol y de adentrarse en un momento nuevo dentro del despliegue del movimiento de la vida.

En este momento, el mundo de lo ente deviene inestable, incluso el cielo y la tierra que presentan en la cotidianidad como polos de referencias absolutos. "Pero plantear una pregunta ante tierra y cielo significa sacrificarse uno mismo para que algo otro pueda ser, para que tierra y cielo no sólo se revelen a sí mismos, sino que se hagan manifestación de algo más alto". ${ }^{35}$ Se revela la existencia auténtica del hombre la cual se abre hacia una trascendencia que se abre más allá del mundo del trabajo y que, también, implica un trans con respecto a la tierra como arraigo originario.

El sacrificio de sí es un despojamiento de todo aquello con lo cual la existencia se ha identificado en el dominio de la función. En este momento, la existencia logra alcanzar su integridad, rompiendo con la lógica de la fragmentación. En la identificación del sí mismo y del otro con el rol, pues, la existencia se atomiza en una serie de recursos empleables. Me convierto y convierto al otro en sus capacidades laborales. Ahora bien, el momento del sacrificio significa un regreso a la unicidad de mí mismo donde, a partir del acto nihilatorio del sacrificio, alcanzo mi

\footnotetext{
32 PATOČKA, El movimiento de la existencia humana, 50.

33 Ibíd., 186.

34 TUCKER, A., "Le sacrifice et l'authenticité : l'éthique de la dissidence tchèque", Revue de théologie et de philosophie Vol 47, 1997, 312.

${ }^{35}$ PATOČKA, El movimiento de la existencia humana, 50.
} 
integridad. Soy yo, como totalidad espiritual, quien se sacrifica, quien realiza la pregunta ante el cielo y la tierra, soy yo quien enfrenta la muerte y el no ser. En ese acto se realiza la asunción de mi propia existencia más allá de mis capacidades y destrezas, más allá de todo rol. La pérdida de sí se reconfigura como encuentro con mi propia integridad, como no divisible, como no atomizable.

Queda de manifiesto que esto no significa un olvido del otro, sino una reconfiguración del sentido de su existencia. La lucha deja de ser y, al no interpretar al otro ya como una fuerza, acontece una recuperación de su integridad. Se produce, entonces, un encuentro efectivo con el otro.

Sólo aquí la dispersión de la vida atomizada es sustituida por una continuidad íntima en la cual el otro no es un extraño sino un yo existente, y que existe ya no abstractamente, en una representación de lo que yo no soy, sino merced a la propia entrega de sí — de modo muy parecido al del amor vital, biológico, pero ahora libre, abierta, universalmente. No es amor como simpatía ni como compasión en el sufrimiento, sino como una misma gloria, una misma victoria: victoria sobre la autodestructiva centración sobre sí mismo. ${ }^{36}$

El tercer momento del movimiento de la vida significa, por tanto, una trasfiguración de la propia existencia como del encuentro con el otro. Si en el primer momento ese otro era arraigo y en el segundo momento una fuerza copartícipe y rival, ahora el otro se muestra en su integridad, como un otro no definido a partir de su rol, sino que, en su desnudez, es descubierto en el modo de alguien a quien me entrego. En este momento del movimiento de la vida, la transmutación de la comprensión del sí mismo hace emerger una nueva forma de comprender al otro signada por el amor. Ese otro a quien me entrego ya no es fuerza empleable sino que recupera su singularidad e integridad.

Siguiendo la tesis de Tucker, el amor se da como modo de relación con el otro. Según el autor, en el momento del sacrificio se "abre la comprensión del Ser de Heidegger para incluir el significado y el amor, [...] para incluir al Dios cristiano y las ideas superiores de Platón en la visión de una edad de oro del regreso al Ser, el fin de la alienación y el establecimiento del amor". ${ }^{37} \mathrm{El}$ amor, pues, acontece como momento disruptivo en la lógica alienante del mundo del rol y la función. Se trata de un amor que no definido por la ausencia sino por una sobreabundancia que proviene de esta pérdida de sí que es, en verdad, un enriquecimiento de sí. La superación auténtica del conflicto es posible, por lo tanto, a partir de esa transfiguración de la existencia que, en su regreso a la verdad, no define al otro a partir de la lucha, sino del amor. Se trata de un amor determinado por la sobreabundancia de una vida que,

\footnotetext{
${ }^{36}$ Ibid., 51.

37 TUCKER, "Le sacrifice et l'authenticité : l'éthique de la dissidence tchèque", 312.
} 
al sacrificarse a sí misma, se enriquece y conquista. Esa conquista es una victoria tanto para la propia existencia como para el otro.

En este sentido, la figura neotestamentaria del agape es, quizá, la que mejor pueda definir aquel amor del que habla el filósofo checo. Cómo señala Ricouer, se trata de un acontecimiento que sobrepasa las lógicas de la reciprocidad y que abre la posibilidad de un reconocimiento que no tiene su fundamento en lógicas utilitarias. El agape es entrega y encuentro con el otro que proviene de la abundancia del corazón ${ }^{38}$.

\section{CONCLUSIÓN}

En el pensamiento de Patočka, el movimiento de la vida se da en una dialéctica donde cada momento es una reconfiguración de ella misma, del mundo, de la tierra y de la relación con los otros. Del arraigo originario a la conquista de sí, el espíritu se transmuta y en esa transmutación lo intersubjetivo adquiere nuevas dimensiones. Lo intersubjetivo es arraigo, luego suelo de lucha a partir de la alienación dada en el mundo del trabajo, y por último conquista de sí.

El planteo de Patočka nos abre a un pensamiento de la existencia donde el encuentro con el otro es una instancia constitutiva en el devenir dialéctico de ella. En este sentido, es interesante señalar la forma en que el filósofo checo logra articular la relación con el otro en sus diversos momentos. A partir de la noción de movimiento, el acontecimiento del arraigo, el acontecimiento de la coparticipación, el acontecimiento de la lucha y el acontecimiento del encuentro auténtico revela una perspectiva que no reduce lo intersubjetivo a un único modo de aparecer.

La oposición entre "ser-con" y "ser contra" se manifiesta como uno de los rasgos del segundo momento del movimiento de la vida. En este sentido, Patočka se distancia igualmente del optimismo ingenuo como del nihilismo. La lucha existe, es real. Sin embargo, es posible de ser superada a partir de una transfiguración de la vida. En esa transfiguración nos encontramos en un estado de apertura a un "más alto" que implica al mismo el encuentro con el otro en una relación libre, signada por el amor, y no por su rol o función. En este sentido, el amor quiebra con la dialéctica ser-con/ser-contra y lleva a un encuentro con el otro más pleno, más auténtico que se da en un habitar en la verdad.

\footnotetext{
${ }^{38}$ Cf. Ricoeur, P., Los caminos del reconocimiento, trad. De Agustín Nogueira, Trotta, Madrid, 2005, 279.
} 